\title{
Experimental and Numerical Characterization of CaPaMan 2bis Operation
}

\author{
E. E. Hernandez-Martinez ${ }^{* 1,2}$, L. Conghui ${ }^{2}$, G. Carbone ${ }^{2}$, M. Ceccarelli ${ }^{2}$, C. S. Lopez-Cajun \\ ${ }^{1}$ Facultad de Ingeniería, Universidad Autónoma de Querétaro, \\ Cerro de las Campanas s/n, 39060 Querétaro, México \\ ${ }^{2}$ Laboratory of Robotics and Mechatronics, DiMSAT, University of Cassino, \\ Via G. Di Blasio 43, 03043 Cassino, Italy. \\ *e.hernandez@unicas.it
}

\begin{abstract}
In this paper, the operation performance of CaPaMan 2bis (Cassino Parallel Manipulator 2 bis) was investigated through lab experimental tests and computer simulations. Milli-CaTraSys (Milli-Cassino Tracking System) was implemented to determine displacements and orientation variations of CaPaMan 2bis end-effector during experimental tests. A 3D (three dimension) virtual model was built in ADAMS environment to simulate the operation behavior for different prescribed motions. Several prescribed motions have been simulated and tested under different conditions in order to characterize the system behavior. In particular, the kinematic characteristics were obtained from both, experimental tests and numerical simulations. Finally, experiment results and simulation computations were compared for purpose of performance evaluation and design characterization of the parallel manipulator structure and its prototype.
\end{abstract}

Keywords: Experimental mechanics, parallel manipulators, simulation, kinematics

\section{RESUMEN}

En este trabajo se investiga el desempeño de la operación del manipulador paralelo CaPaMan 2 bis a través de pruebas experimentales y de simulaciones numéricas. El sistema Milli-CaTraSys se implementó para determinar la variación de los desplazamientos y la orientación del órgano terminal de CaPaMan 2bis durante las pruebas experimentales. Un modelo 3D desarrollado en ADAMS fue utilizado para simular el comportamiento operacional para diferentes movimientos prescritos. Tales movimientos han sido simulados y verificados bajo diferentes condiciones con el fin de caracterizar el comportamiento del sistema. En particular, se obtienen características cinemáticas de las pruebas experimentales y de las simulaciones numéricas. Finalmente, se comparan los resultados experimentales con los cálculos de simulación con el propósito de evaluar el desempeño y la caracterización del diseño del manipulador paralelo y su prototipo.

\section{Introduction}

Parallel manipulators are well understood for higher payload capability, stiffness, accuracy and dynamic performance in contrast to traditional serial manipulators $[1,2]$. A parallel manipulator has the main property of having its gripper connected to the base through several serial (and/or parallel) kinematic chains. Nowadays, some commercial parallel robots are already available in the market for different applications such as packaging, materials handling and machining [3]-[5]. Nevertheless, the parallel robots field has attracted research interests for the last two decades and many prototypes have been built in laboratories for academic research. As a consequence, the number of works that deal with open problems of this mechanism type has also grown. For example, an extensive survey on commercial applications and state of the art of parallel robots can be found in the web page [6]. In fact, problems of parallel robots are still open for efficient exploitation of their inherent advantages, mainly small workspace, and complexity of direct kinematic equations or poor dexterity [7]. For this reason, it can be interesting to study mechanisms with a minor mobility that are able to perform elementary motions, namely translation, rotation 
or planar movements. In addition, many applications can be achieved with less than six degree of freedom (DOF). Indeed, a three DOF mechanism could be enough, thus reducing the total cost and operations for end-effector positioning.

At LARM: Laboratory of Robotics and Mechatronics, University of Cassino, several prototypes of parallel manipulators have been built with low-cost and easy-operation characteristics. Research activities have been carried out on both theoretical study and application aspects. A three DOF spatial parallel manipulator named as CaPaMan has been proposed with peculiar mechanism architecture [8]. This parallel manipulator has been studied to analyze its architecture in terms of workspace, kinematic formulation, optimal design, stiffness properties and dynamic performance, [2] [9]-[12]. In addition, the platform of CaPaMan has been used as a motion generator for earthquake simulation [13]. Later, a new version called CaPaMan 2 has been conceived and built at LARM. Each prismatic joint on the coupler for each CaPaMan leg has been substituted by a revolute joint. Afterwards, CaPaMan 2 has been aimed for drilling applications, and then stiffness and accuracy have been improved by paying special attention to mechanical design. CaPaMan 2 bis has been the result of these enhancements, where the modified shape of the movable plate has been designed to properly lock a commercially available drilling tool. A prototype of CaPaMan 2bis built at LARM is shown in Figure 1(a). It has been implemented as a part of a hybrid robotic architecture for surgical tasks in the work [14], as well as a trunk module in a humanoid robot design named as CALUMA (CAssino Low-cost hUMAnoid robot) [15]. Previous works were developed to analyze the CaPaMan 2bis prototype in terms of stiffness and basic kinematics $[16,17]$. Since the solution of direct kinematic problem for CaPaMan 2 bis can be complex, the system behavior can be conveniently simulated and experimentally measured.

In this paper, operational performances of CaPaMan 2bis are investigated both in experimental tests and simulation. Milli-CaTrays, a measuring system built at LARM was implemented to measure the position and orientation of the end-effector on the platform of CaPaMan 2 bis. Several motions with different conditions were prescribed for characterizing the system behavior. Simulation was carried out in ADAMS environment in order to investigate the feasibility of this parallel robot. Experiment results and simulation computations were compared for the purpose of operation performance evaluation and design characterization of the architecture design and its prototype.

\section{Parallel manipulator design}

A kinematic scheme of CaPaMan 2 bis is shown in Figure $1(b)$. It is composed of a movable platform (MP) connected to a fixed base (FP) through three leg mechanisms. Each leg mechanism is composed of an articulated parallelogram (AP) whose coupler carries a revolute joint (RJ), a connecting bar (CB) that transmits the motion from AP to MP through $\mathrm{RJ}$, and a spherical joint (BJ), which is installed on MP at point J. Revolute joint RJ installed on the coupler of AP has the rotation axis coinciding with the parallelogram plane. Each leg mechanism is rotated $2 \pi / 3$ with respect to the neighboring one so that the leg planes lie along two vertices of an equilateral triangle, giving symmetry properties to the mechanism. Design parameters of the mechanism are shown in Figure 1(b). They can be identified for the $k$-th leg mechanism $(k=1,2,3)$ as: $a_{k}$ is the length of the frame link; $b_{k}$ is the length of the input crank; $c_{k}$ is the length of the coupler link; $d_{k}$ is the length of the follower crank and $h_{k}$ is the length of the connecting bar. The size of movable platform MP and fixed base FP are given by distances $r_{p}$ and $r_{f}$, as shown in Figure 1(b). Points 
$H$ and $O$ are the center points of MP and FP, respectively. Points $O_{k}$ are the middle point of frame link $a_{k}, J_{k}$ are the connecting points between the $k$-th leg mechanism and platform MP. The design parameters of the CaPaMan 2bis are listed in Table I.

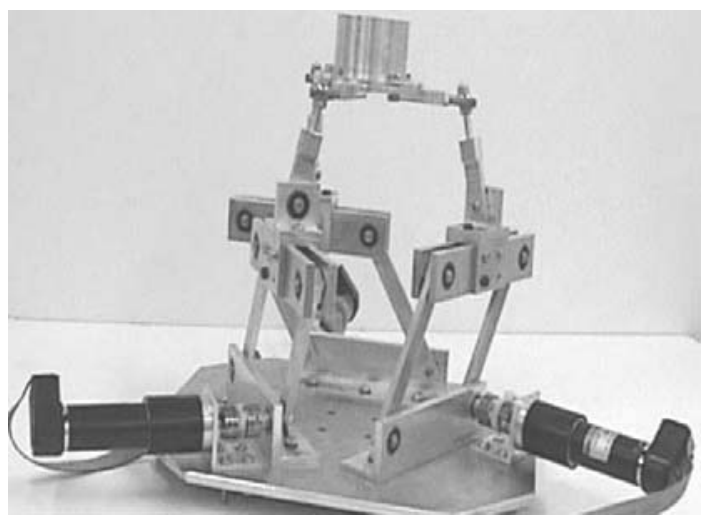

(a)

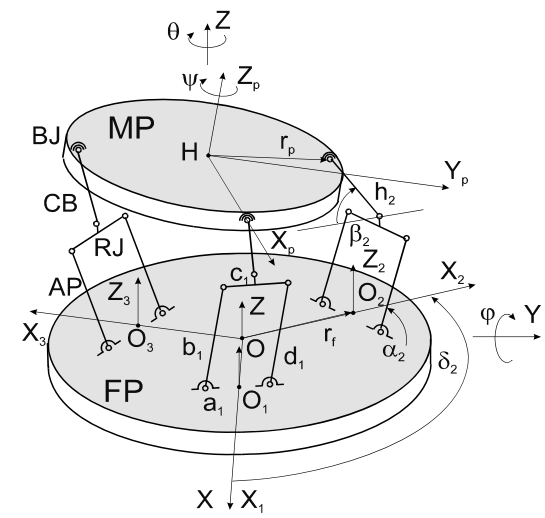

(b)

Figure 1. Cassino Parallel Manipulator 2bis: (a) A prototype built at LARM; (b) A kinematic scheme of CaPaMan 2 bis.

\begin{tabular}{|c|c|c|c|c|c|}
\hline$a_{k}=c_{k}[\mathrm{~mm}]$ & $b_{k}=d_{k}[\mathrm{~mm}]$ & $r_{p}[\mathrm{~mm}]$ & $r_{f}[\mathrm{~mm}]$ & $h_{k}[\mathrm{~mm}]$ & $I_{c x y}=I_{c x y}=I_{c y z}\left[\mathrm{~kg} \cdot \mathrm{mm}^{2}\right]$ \\
\hline 100 & 100 & 65 & 65 & 50 & 0 \\
\hline$\alpha_{k}[\mathrm{deg}]$ & $\beta_{\mathrm{k}}[\mathrm{deg}]$ & $m[\mathrm{~kg}]$ & $\tau[\mathrm{N} / \mathrm{m}]$ & $I_{c z z}\left[\mathrm{~kg} \cdot \mathrm{mm}^{2}\right]$ & $I_{c x x}=I_{c x y}\left[\mathrm{~kg} \cdot \mathrm{mm}^{2}\right]$ \\
\hline 45 to 135 & 30 to 120 & 2.3 & 2 & 24600 & 12400 \\
\hline
\end{tabular}

Table 1. Mechanical design parameters and inertial properties of CaPaMan 2 bis.

An inertial frame $O-X Y Z$ has been assumed to be fixed to base FP. A moving frame $H X_{P} Y_{p} Z_{p}$ has been attached to platform MP. O-XYZ has been fixed with $Z$ axis orthogonal to the FP plane and $X$ axis as coincide with the line joining $O$ to $O_{1}$. Moving frame $H X_{P} Y_{P} Z_{P}$ has been fixed to platform MP with $Z_{p}$ orthogonal to the MP plane and $X_{P}$ axis as coincident to the line joining $H$ to $J$ and $Y_{P}$ to give a Cartesian frame. Angle $\delta_{k}$ is the structure rotation angle between $O X_{1}$ and $O X_{k}$ as well as between $H J_{1}$ and $H J_{k}$. They are equal to $\delta_{1}=0$, $\delta_{2}=2 \pi / 3$ and $\delta_{3}=4 \pi / 3$.
The position of $H$ in the center of movable platform MP is represented by coordinates $x, y$ and $z$. The orientation of MP with respect to fixed base FP can be represented with the three Euler angles $\theta, \varphi$ and $\psi$ as shown in Fig. 1(b), where $\theta$ is a rotation about the $Z$ axis; $\varphi$ is a tilting rotation about the $Y_{P}$ axis, which is the $Y$ axis after a $\theta$ rotation; and $\psi$ is a rotation about the $Z_{p}$ axis. For each leg mechanism, the kinematic variables are input crank angles $\alpha_{k}$ and passive revolute angle $\beta_{k}$, which is between the connecting bar and axis $\mathrm{Xk}$. Therefore, each leg mechanism has two 
degrees of freedom. Thus, three legs have six degrees of mobility and the movable platform has additionally six degrees of mobility. But since each spherical joint connecting the movable platform and leg mechanism gives three constraints, thus the degrees of mobility of CaPaMan 2 bis can be computed as $n=(3 * 3+6)-3 * 3=3$.

As shown in Fig. 1(b), if one refers to a single leg, the coordinates of the center of the k-th ball joint can be written as

$x_{k}=h_{k} \cos \beta_{k}$

$y_{k}=b_{k} \cos \alpha_{k}$

$\mathrm{z}_{\mathrm{k}}=\mathrm{b}_{\mathrm{k}} \sin \alpha_{\mathrm{k}}+\mathrm{h}_{\mathrm{k}} \sin \beta_{\mathrm{k}}$

Position and orientation of the platform can be obtained in closed-form through the expressions [6]

$x=\frac{y_{3}-y_{2}}{\sqrt{3}}-\frac{r_{p}}{2}(1-\sin \varphi) \cos (\psi-\theta)$

$y=y_{1}-r_{p}(\sin \psi \cos \theta+\cos \psi \sin \varphi \sin \theta)$

$z=\frac{z_{1}+z_{2}+z_{3}}{3}$

$\theta=\sin ^{-1}\left[2 \frac{y_{1}+y_{2}+y_{3}}{3 r_{p}(1+\sin \varphi)}\right]-\psi$

$\psi=\tan ^{-1}\left(\sqrt{3} \frac{z_{3}-z_{2}}{2 z_{1}-z_{2}-z_{3}}\right)$

$\varphi=\cos ^{-1}\left( \pm \frac{2}{3 r_{p}} \sqrt{z_{1}^{2}+z_{2}^{2}+z_{3}^{2}-z_{1} z_{2}-z_{2} z_{3}-z_{1} z_{3}}\right)$ with

$\left(z \geq z_{1} \Rightarrow "+" ; z<z_{1} \Rightarrow "-"\right)$

where coordinates $y_{k}$ and $y_{k}$ are given by Equations (2) and (3). However, coordinates yk and $z_{k}$ cannot be computed directly since the passive revolute angles $\beta_{\mathrm{k}}$. The solution of the direct kinematic problem for CaPaMan 2bis can be very complex and closed-form algebraic Equations (4)-(9) cannot be used as in CaPaMan [17].

\section{Experimental setup}

As explained above, regarding CaPaMan 2bis, it was very difficult to obtain a closed-form kinematic formulation. Thus, a suitable measuring system was used to measure the end-effector pose of CaPaMan 2 bis. Recently, a measuring system was developed at LARM, whose name is Milli-CaTraSys $[16,18,19]$. It is a cable-based architecture which can be used to measure the position and orientation of the end-effector of robotic systems. The Milli-CaTraSys design is based on a parallel manipulator configuration with 3-2-1 GoughStewart architecture. The kinematics of this type of measuring system has been solved in a closedform as a result of its special architecture by applying the trilateration technique. Moreover, Milli-CaTraSys can be used to apply a known wrench on the end-effector of a robotic system and, simultaneously, to measure the corresponding end-effector displacements. Since it uses known masses $m_{i}$ (with $i=1, \ldots, 6$ ) on the free ends of its cables, one can use Milli-CaTraSys for analyzing stiffness features of robotic systems. It has been already used successfully for evaluation of stiffness of robots as reported in [16]. MilliCaTraSys consists of a fixed platform and a specific design end-effector with six cables. A set of six LVDT transducers is fixed at the end of each cable to determine the length change. The data acquisition and elaboration are executed by a NI DAQ data acquisition PCl 6024 card and a virtual instrument program in LABVIEW environment. A setup of the Milli-CaTraSys tracking system for measuring the motion of CaPaMan 2bis is shown in Figure 2. The six cables of Milli-CaTrasys are fixed on a specific designed end-effector that is installed on the movable platform MP of CaPaMan 2bis. 
Three fixed points on the movable platform MP define a triangle on the end-effector.

A scheme of the experimental set-up for pose measuring of CaPaMa2 bis during a lab test at LARM is shown in Figure 3. In general, the experimental system consisted of two parts: the CaPaMan 2 bis system and Milli-CaTraSys tracking system. The motion control system of prototype CaPaMan 2 bis consists of a mechanical prototype as shown in Figure 1(a), whose leg mechanisms are actuated by Maxon RE25 DC motors, a control unit with the amplifiers of motors, and a $\mathrm{NI}$ data acquisition card. A program was developed in the LABVIEW environment to give control instructions and to handle the acquired data.

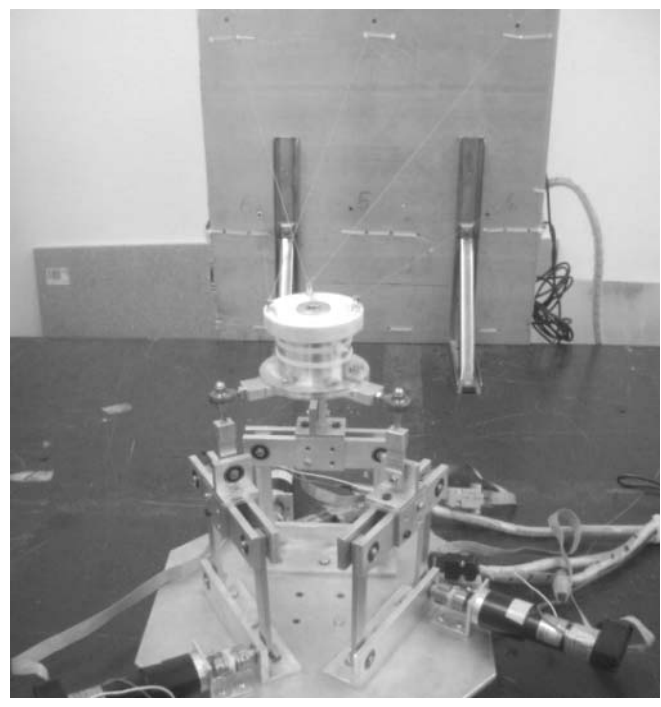

Figure 2. Experimental setup by using Milli-CaTraSys tracking system.
By measuring the length of the cables and using trilateration technique, the position and orientation of the Milli-CaTraSys end-effector can be determined in Cartesian space. This technique is based on suitable algebraic manipulations of distances $I_{i}$ as function of the Cartesian coordinates $X_{H}, y_{H}$ and $z_{H}$ of a point $H$ with respect to the fixed frame $0-X Y Z$ in the form

$$
\begin{gathered}
l_{i}^{2}=\left(x_{H}-x_{B i}\right)^{2}+\left(y_{H}-y_{B i}\right)^{2}+\left(z_{H}-z_{B i}\right)^{2} \\
(\text { with } i=1,3,5)
\end{gathered}
$$

where $X_{B i}, Y_{B i}$ and $Z_{B i}$ are the Cartesian coordinates of fixed points $B_{i}$ at the Milli-CaTraSys base, as shown in Figure 4(a). After some algebraic manipulation, the position of $H$ can be computed through its components as

$$
\begin{aligned}
& x_{H}=H_{x}\left(-B_{2}-\sqrt{B_{2}^{2}-4 B_{1} B_{3}}\right) / 2 B_{1}+E_{x} \\
& y_{H}=H_{y}\left(-B_{2}-\sqrt{B_{2}^{2}-4 B_{1} B_{3}}\right) / 2 B_{1}+E_{y} \\
& z_{H}=\left(-B_{2}-\sqrt{B_{2}^{2}-4 B_{1} B_{3}}\right) / 2 B_{1}
\end{aligned}
$$

where

$$
\begin{aligned}
& B_{1}=H_{x}^{2}+H_{y}^{2}+1 \\
& B_{2}=2 H_{x}\left(E_{x}-x_{3}\right)+2 H_{y}\left(E_{y}-y_{3}\right)-2 z_{3} \\
& B_{3}=E_{x}^{2}+E_{y}^{2}+A_{3}^{2}-2 E_{x} x_{3}-2 E_{y} y_{3} B_{1} \\
& H_{x}=-\left(Z_{21}+H_{y} Y_{21}\right) / X_{21} \\
& E_{x}=\left(A_{1}+A_{2}-E_{y} Y_{21}\right) / X_{21} \\
& E_{y}=\frac{-A_{1}+A_{2}}{Y_{32} X_{21}}
\end{aligned}
$$

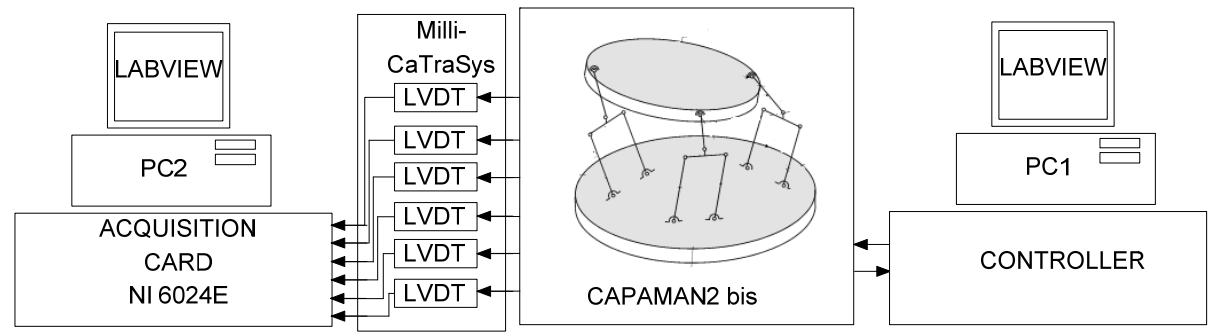

Figure 3. A scheme of the setup for the experimental tests of CaPaMan 2bis and Milli-CaTraSys at LARM. 
with $(i, j=1,2,3),(i \neq j)$

$A_{i}=l_{i}^{2}-x_{i}^{2}-y_{i}^{2}-z_{i}^{2}$

$X_{i j}=x_{i}-x_{j}$

$Y_{i j}=y_{i}-y_{j}$

$z_{i j}=z_{i}-z_{j}$

Equations (11) and (12) express a general formulation of the trilateration scheme of Figure 4(a). Using expressions similar to Equations (11) to (13) for measured lengths $I_{2}$ and $I_{6}$ and $H F$ distance, it is possible to determine the position of point $F$ through its components $X_{F}, y_{F}$ and $z_{F}$. Afterwards, the orientation of segment $H F$ can be usefully described in the space through angles $\alpha, \beta$ and $\psi$, which can be obtained from the geometry of Figure 4(b) as

$$
\begin{aligned}
& \alpha=\tan ^{-1}\left(\frac{y_{H}-y_{F}}{x_{H}-x_{F}}\right) \\
& \beta=\tan ^{-1}\left(\frac{z_{H}-z_{F}}{\left(\left(y_{H}-y_{F}\right)^{2}+\left(x_{H}-x_{F}\right)\right)^{1 / 2}}\right) \\
& \psi=\tan ^{-1}\left(\frac{z_{Q}-z_{C}}{\sqrt{\left(y_{Q}-y_{C}\right)^{2}}+\left(x_{Q}-x_{C}\right)^{2}}\right)
\end{aligned}
$$

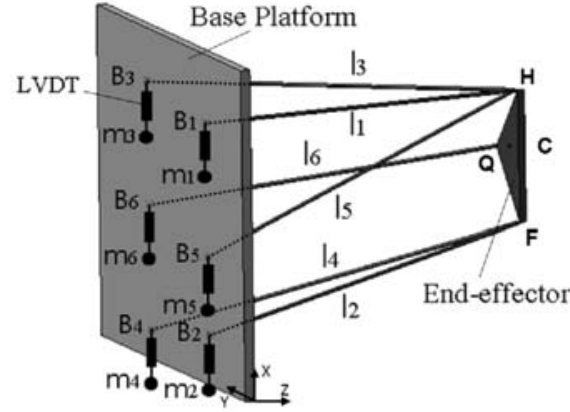

(a)
Calibration work is needed both for CaPaMan 2bis and Milli-CaTraSys to have accuracy in control and measurement. A calibration process was carried out in order to determine a suitable initial configuration for the measuring system as well as to verify and to reduce the effects of systematic errors on its accuracy. During calibration, prototype CaPaMan 2bis is installed on a fixed rigid platform with base FP orthogonal with the platform of Milli-CaTraSys as shown in Figure 2. The position of point $O$ of base FP can be easily measured with respect to an inertial reference frame fixed on the platform of Milli-CaTraSys.

The calibration and experimental tests of MilliCaTraSys were carried out with LVDT B type transducers with a range of $100 \mathrm{~mm}$. The calibration procedure can be summarized as in the flowchart of Figure 5, where $\varepsilon_{j}$ and $\delta_{i}$ are acceptable calibration errors prescribed by the user. Depending on the required accuracy level, these values can be minimized even in an iterative process. Firstly, during the calibration procedure, each cable was placed in an initial reference position. A virtual instrument in the LabVIEW environment was developed for acquisition and processing of the data from the amplifiers of the LVDTs. The characteristic curve voltage-length of each LVDT can be defined by a line with its $Y$-axis

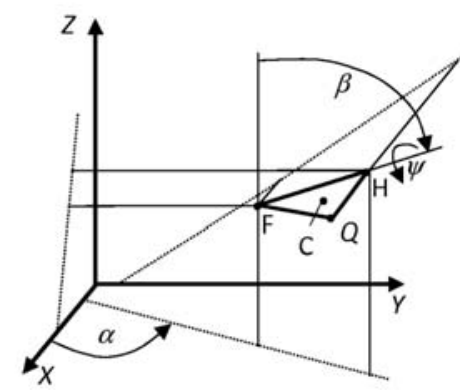

(b)

Figure 4. Schemes for Milli-CaTraSys system: (a) A 3D scheme of Milli-CaTraSys with the reference frame;

(b) A scheme showing the end-effector orientation through set angles $\alpha, \beta$ and $\psi$. 
intersection and scope. In the calibration procedure, these values are defined as the SUM Block value and the MULT Block value in the virtual instrument, respectively. Also, it is necessary to adjust the voltage offset as prescribed in the LVDT characteristic curve. Individual calibration of each LVDT was achieved by displacing the cables to know distances $I_{R i}$. Calibration error $\delta_{i}$ is defined by the difference between measured distance $I_{M i}$ and known distance $I_{R i}$.



Figure 5. A flowchart of the calibration procedure for Milli-CaTraSys

\section{Simulation model}

In addition to experimental tests, in this work a simulation of CaPaMan 2bis and Milli-CaTraSys was carried out in a numerical way. Thus, a 3D model of CaPaMan 2bis and Milli-CaTraSys was built in the ADAMS dynamic simulation environment. ADAMS is powerful software used successfully to simulate the dynamic behavior of vehicles $[20,21]$ and sometimes to design parallel kinematic systems [22]. By using this software, the kinematic and dynamic operation performances of CaPaMan 2 bis was investigated through a proper model, as shown in Figure 6 . The design parameters used for the 3D model of CaPaMan 2bis are those of the built prototype, and listed in Table I. Milli-CaTraSys cables were modeled by means of a set of six forces between the fixed points on the MilliCaTraSys base and the attachment points on platform MP of CaPaMan 2bis. As shown in Figure 6 , the total torque applied on the CaPaMan 2bis end-effector by the effect of these forces is modeled by means of a proper torque measured in the center of platform MP of CaPaMan 2bis.

Once the model of CaPaMan 2bis is properly adjusted in the ADAMS environment, different input motions were prescribed for the three input crank angles $\alpha_{k}$ (with $k=1,2,3$ ) to simulate different motions of the platform. Particularly, the same input motions were also used during the experimental tests. Table II lists the prescribed motions used for input crank angles $\alpha \mathrm{k}$ during simulation.

As shown in Table II, three different input motions were prescribed for the simulation. For each case of study, the starting and end values of input crank angles $\alpha_{k}$ (with $\mathrm{k}=1,2,3$ ), as well as actuation time $t_{k}$ (with $k=1,2,3$ ) were given. Actually, since the three Maxon DC motors in the prototype of CaPaMan 2 bis provide a position and velocity closed-loop algorithm control with a high dynamic performance, a cubic function was used to produce the input reference angles for each cycle as [9]

$$
\alpha_{\mathrm{k}}=c_{0}+c_{1} t+c_{2} t^{2}+c_{3} t^{3}
$$




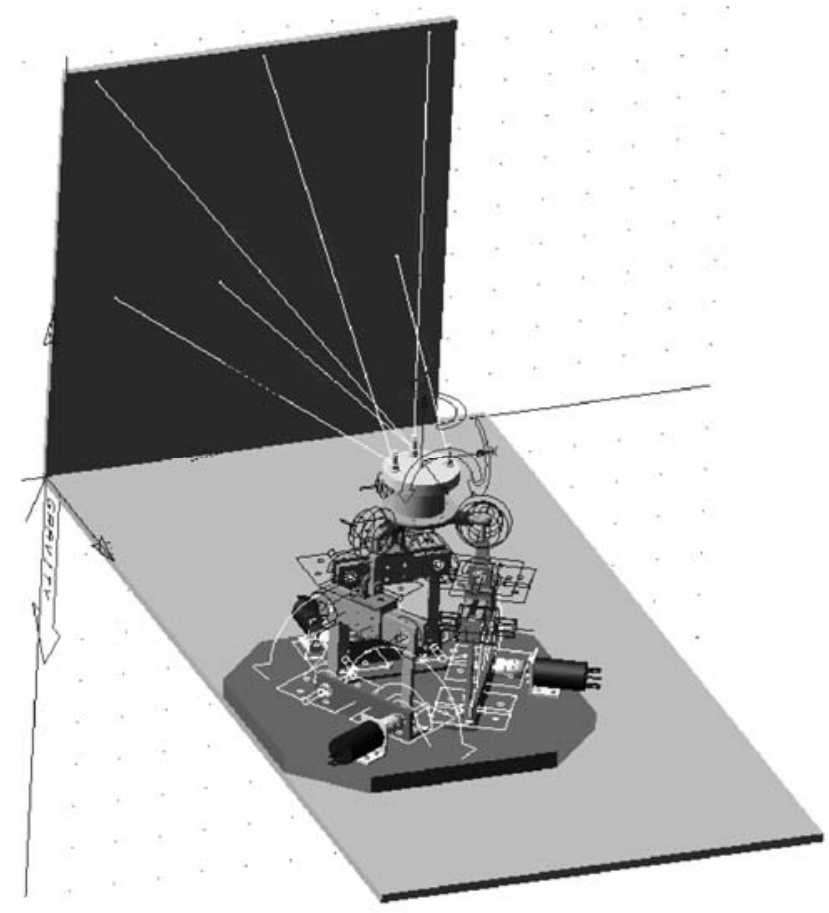

Figure 6. A 3D model of CaPaMan 2bis and Milli-CaTraSys built in ADAMS environment.

where four unknown constant values $c_{i}$ (with $\mathrm{i}=0$, 2,3 ) in Equation (15) can be determined by solving four constraint equations. These are given by the values of position and velocity at the starting and end points. In each simulated case, position and orientation ofplatform MP was computed in the simulation environment by selecting proper reference frames and markers. Moreover, proper
ADAMS functions were built to measure correctly the position and orientation of CaPaMan 2bis MP platform in simulations. In particular, orientation functions were prescribed as in Equation (14). Additionally, the torque for each motor can also be determined to characterize the feasibility of the prescribed motion for the prototype of CaPaMan 2bis.

\begin{tabular}{|c|c|c|c|c|c|c|c|c|c|}
\hline \multirow{2}{*}{ Motion: } & \multicolumn{10}{|c|}{ Conditions } \\
\cline { 2 - 11 } & $\alpha_{1 \mathrm{i}}$ & $\alpha_{1 \mathrm{f}}$ & $t_{1}$ & $\alpha_{2 \mathrm{i}}$ & $\alpha_{2 \mathrm{f}}$ & $t_{2}$ & $\alpha_{3 \mathrm{i}}$ & $\alpha_{3 \mathrm{f}}$ & $t_{3}$ \\
\hline Three legs & 78 & 102 & 4 & 78 & 102 & 4 & 78 & 102 & 4 \\
\hline Two legs & 80 & 95 & 4 & 100 & 85 & 4 & 90 & 90 & 4 \\
\hline One Leg & 78 & 108 & 4 & 90 & 90 & 4 & 90 & 90 & 4 \\
\hline
\end{tabular}

Table 2. Parameters for different input motions in simulation. 


\section{Analysis of simulation and experimental results}

In this section, for each case of study listed in Table II, the position of end-effector center point $C$ and orientation angles of the MP platform have been reported both for experiment results and computer simulation. In Figures (7)-(9), results are reported for the first case in Table II. The same motion was prescribed for the three legs of CaPaMan 2 bis. The simulated motion starts from point $a_{s}$, goes to $b_{s}$ and ends at point $c_{s}$, as well as the measured motion starts from $a_{e}$, goes to $b_{e}$ and ends at point $c_{e}$. In one cycle of motion, Figure 7(a), the differences between curves $a_{e}-b_{e}$ and $b_{e^{-}}$ $c_{e}$ could be due to the backlash and clearances in the mechanism of the prototype. As shown in Figure 7 (b), (c) and (d) the displacement is $2 \mathrm{~mm}$ in $X$ axis, $1 \mathrm{~mm}$ in $Y$ axis and $5.5 \mathrm{~mm}$ in $Z$ axis, respectively. In Figure 8(a), three orientation angles $\phi, \theta$ and $\psi$ are shown in 3D space.

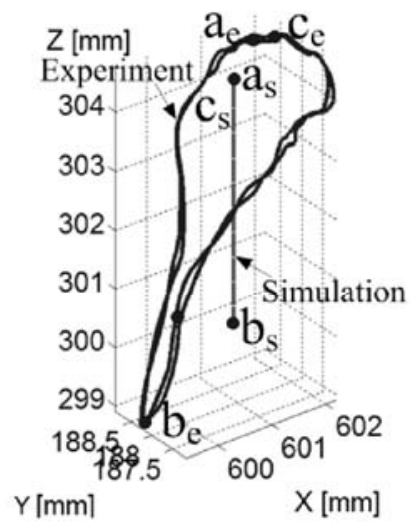

(a)

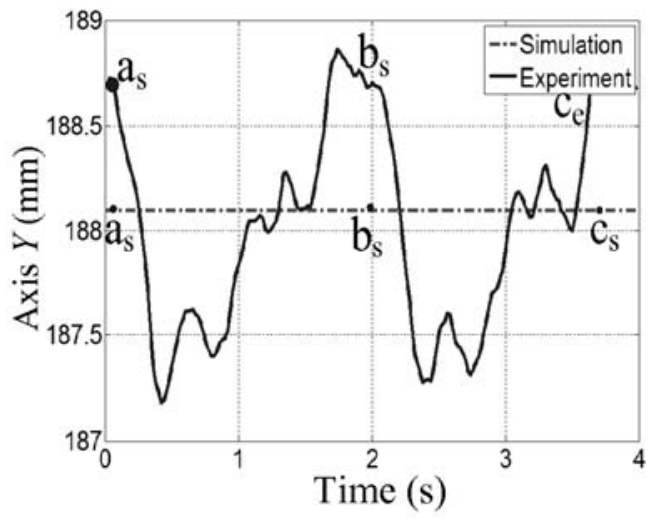

(c)

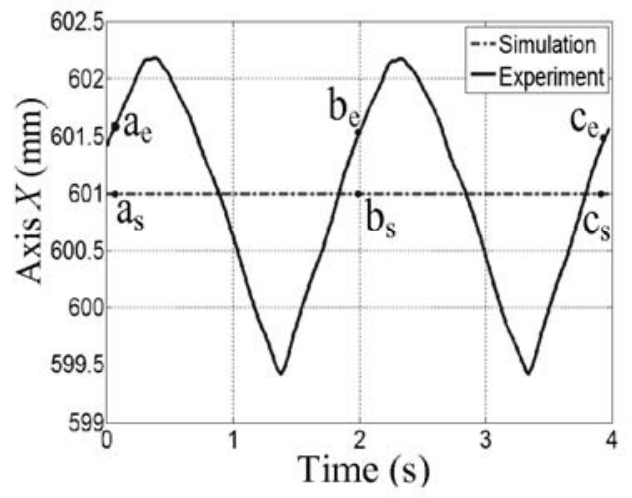

(b)

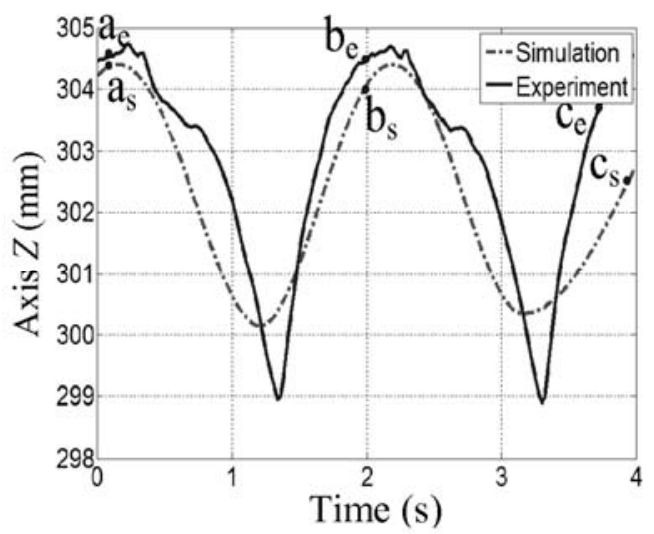

(d)

Figure 7. Position of platform MP for three-legged motion: (a) Position of point $\mathrm{H}$ in Cartesian space; (b) Position in the $X$ axis; (c) Position in the $Y$ axis; (d) Position in the $Z$ axis 
In Figures 8(b), (c) and (d), the orientation angles are plotted with respect to time. The maximum position difference is $2 \mathrm{~mm}$ as shown in Figure 9(a). The differences could be due to several

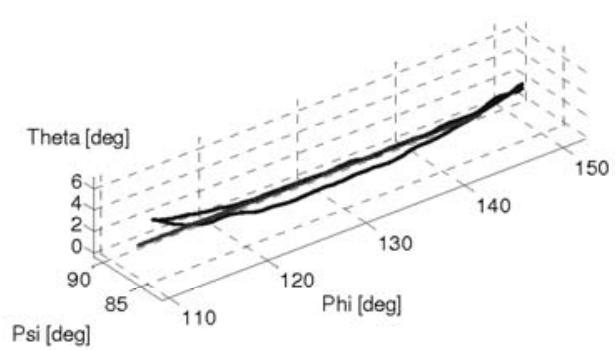

(a)

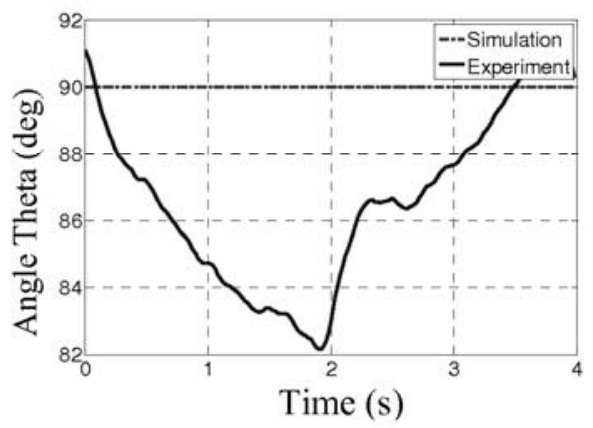

(c) reasons, among them, errors in ADAMS modeling and computation, calibration errors in the experimental setup, and errors in the control algorithm in the CaPaMan 2bis system.

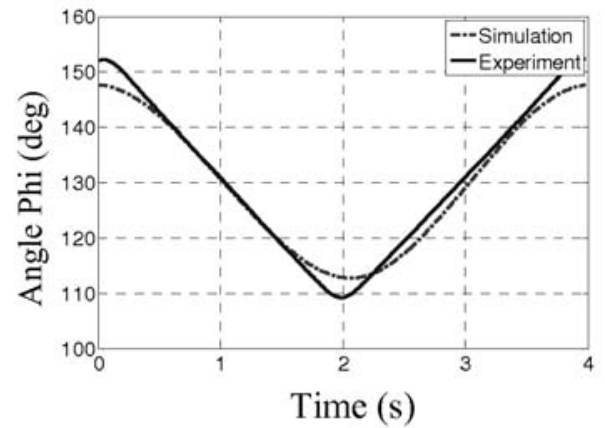

(b)

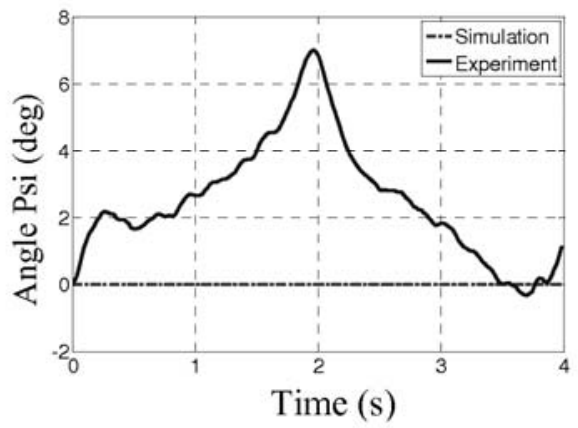

(d)

Figure 8. Orientation of platform MP for three-legged motion: (a) A view in 3D space; (b) Angle $\phi$; (c) Angle $\theta$; (d) Angle $\psi$.

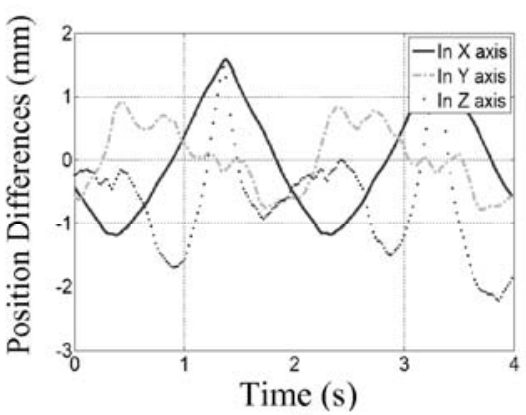

(a)

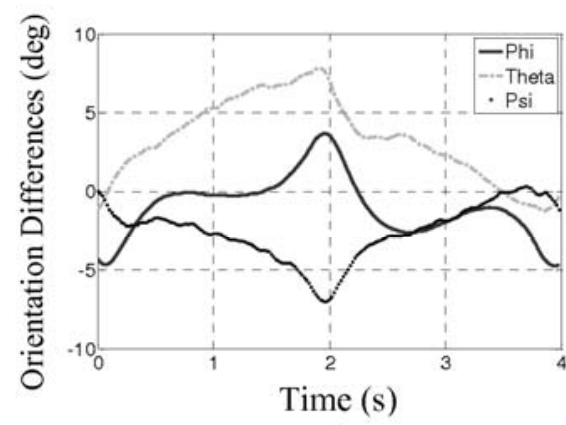

(b)

Figure 9. Differences between simulation and experimental results for three-legged motion:

(a) Position differences; (b) Orientation angles differences 
In four cycles of motion, Figures 10 (a) and (b) show the position and orientation repeatability for experiments with three legs of CaPaMan 2bis. The motion of the three legs of CaPaMan 2bis from -12 to $12 \mathrm{deg}$. provides as output a maximum motion for point $C$ on the movable plate of about $5.5 \mathrm{~mm}$ in $Z$ direction with a maximum rotation $\phi$ about the Z-axis of $42 \mathrm{deg}$. as shown in the plots of Figures $7(\mathrm{~d})$ and $8(\mathrm{~b})$, respectively. Table III summarizes the maximum movable ranges for the three prescribed motions of CaPaMan 2bis described in Table II. For three-legged motion, the prototype performs a quasi translation motion along the $\mathrm{Z}$ axis with a rotation in angle $\phi$. Therefore, it can be stated that the prototype of CaPaMan 2 bis shows a good ability to run accurately operations with prescribed motion characteristics. From the above results, a more accurate operation can be expected by improving calibration and experimental setup.

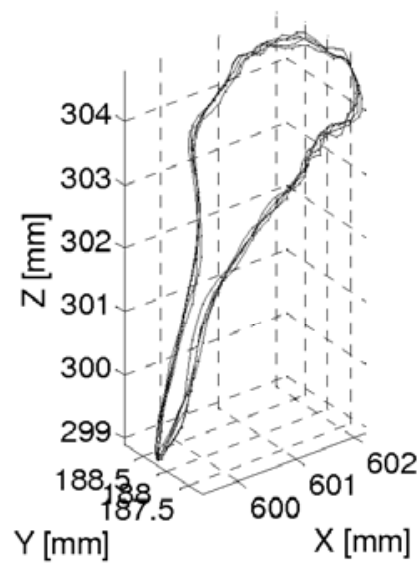

(a)

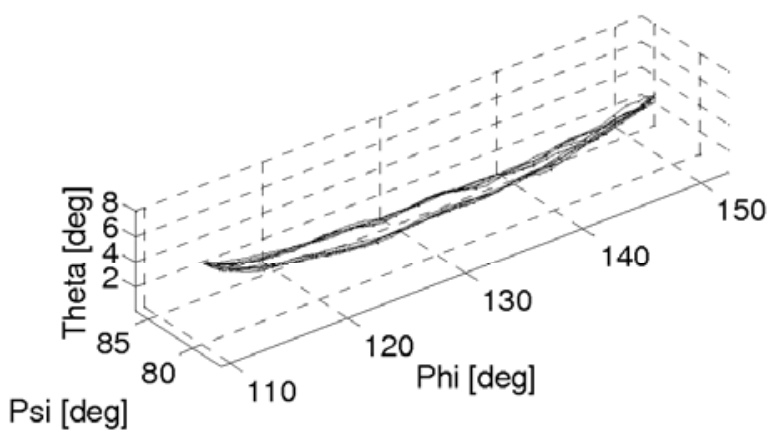

(b)

Figure 10. Experimental repeatability for four cycles of motion with three legs of CaPaMan 2bis: (a) Position of point $\mathrm{H}$ in Cartesian space; (b) Orientation of the MP platform in 3D space

\begin{tabular}{|c|c|c|c|c|c|c|c|c|c|c|c|c|}
\hline & \multicolumn{2}{|c|}{$X_{H}[\mathrm{~mm}]$} & \multicolumn{2}{|c|}{$Y_{H}[\mathrm{~mm}]$} & \multicolumn{2}{|c|}{$Z_{H}[\mathrm{~mm}]$} & \multicolumn{2}{|c|}{$\phi[\mathrm{deg}]$} & \multicolumn{2}{|c|}{$\theta$ [deg] } & \multicolumn{2}{|c|}{$\psi$ [deg] } \\
\hline & Min & Max & Min & Max & Min & Max & Min & Max & Min & Max & Min & Max \\
\hline $\begin{array}{c}\text { Three } \\
\text { legs }\end{array}$ & 599.5 & 602.25 & 187.25 & 188.75 & 299 & 304.5 & 110 & 152 & 82 & 91 & 0 & 7 \\
\hline Two legs & 580 & 610 & 175.80 & 179.50 & 297.6 & 304.3 & 128.5 & 132.4 & 89 & 92.7 & -3 & 5 \\
\hline One leg & 581 & 517 & 171 & 187 & 297.5 & 304 & 118 & 137.5 & 88.2 & 93.3 & -4.2 & 3 \\
\hline
\end{tabular}

Table 3. Measured maximum and minimum reaches for the three motions of CaPaMan 2bis 


\section{Experimental and Numerical Characterization of CaPaMan 2bis Operation, E. E. Hernandez et al., 101-119}

In Figures (11)-(13), results are reported for the second case of Table II. As shown in Figure 11, for the experiment results, the displacement is $30 \mathrm{~mm}$ in the $X$ axis, $3.6 \mathrm{~mm}$ in the $Y$ axis and $6.5 \mathrm{~mm}$ in the $Z$ axis. The maximum position difference is 6 $\mathrm{mm}$ as shown in Figure 13(a). The three orientation angles are plotted in Figure 12.

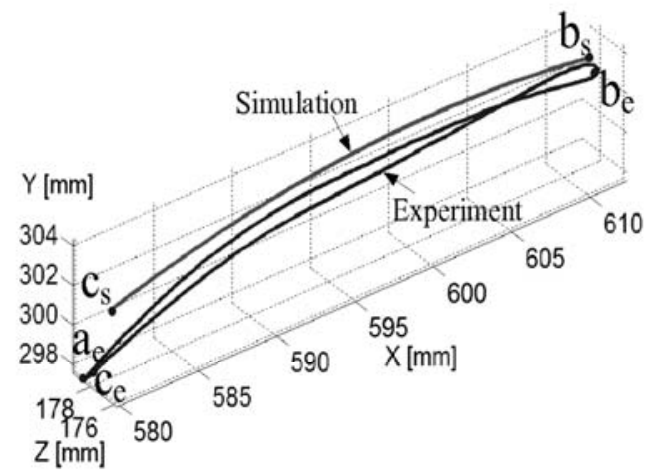

(a)

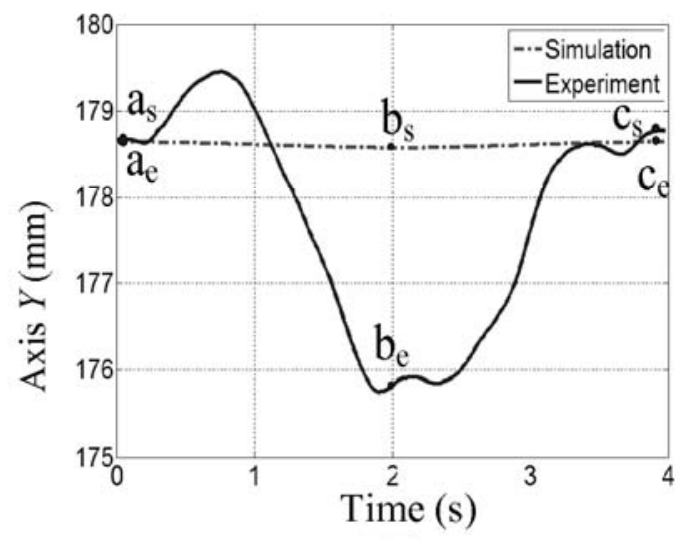

(c)
Orientation angles $\phi, \theta$ and $\psi$ are near 130, 90 and 0 degrees, respectively. The maximum orientation difference is 4.8 degrees as shown in Figure 13(b). In four cycles of motion, Figures 14 (a) and (b) show the position and orientation repeatability for experiments with two legs of CaPaMan 2bis. Therefore, a quasi translation motion along the $X$ axis has been obtained when two legs move.

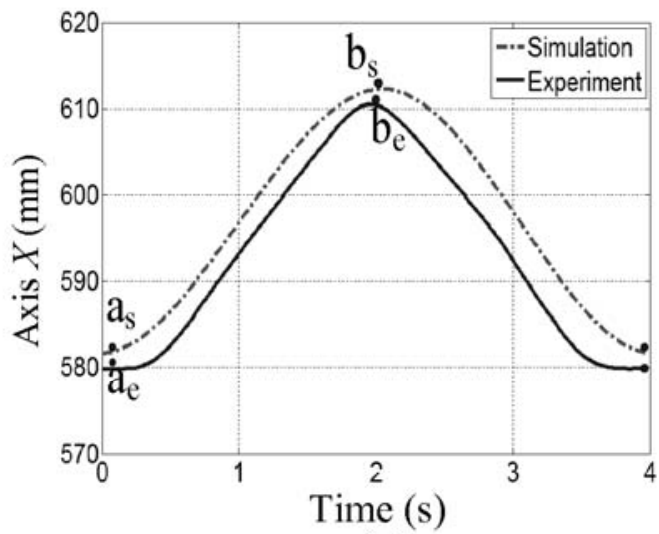

(b)

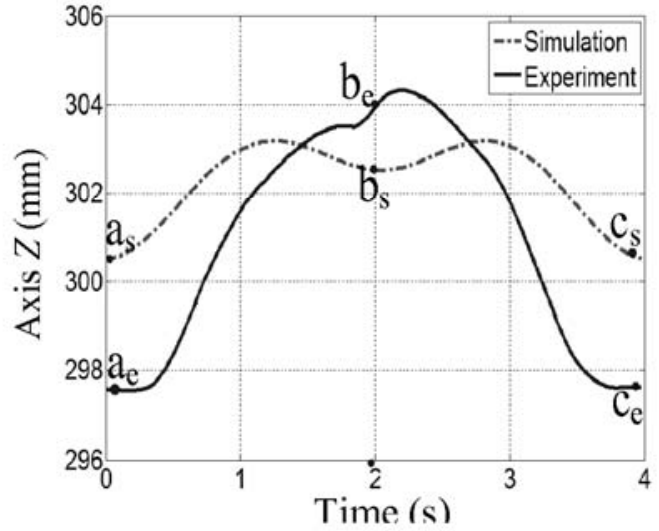

(d)

Figure 11. Position of platform MP for two-legged motion: (a) Position of point $H$ in Cartesian space; (b) Position in the $X$ axis; (c) Position in the $Y$ axis; (d) Position in the $Z$ axis 


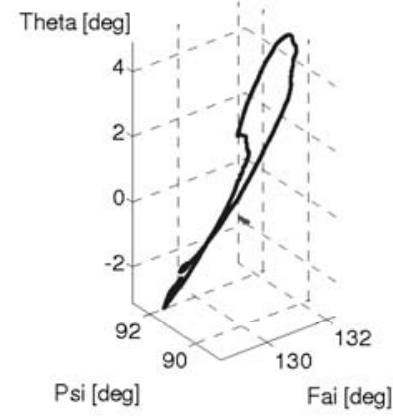

(a)

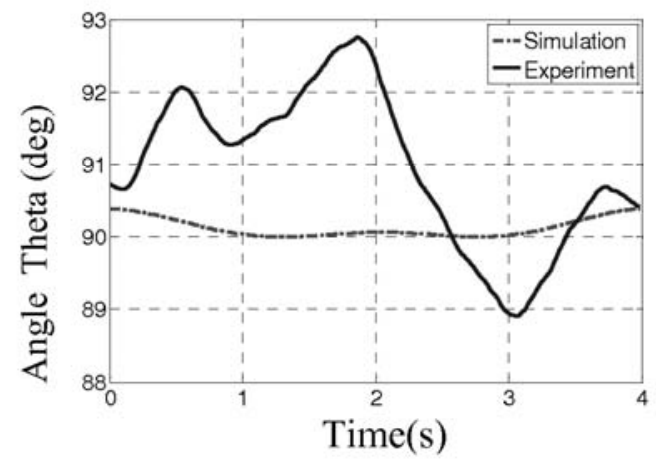

(c)



(b)

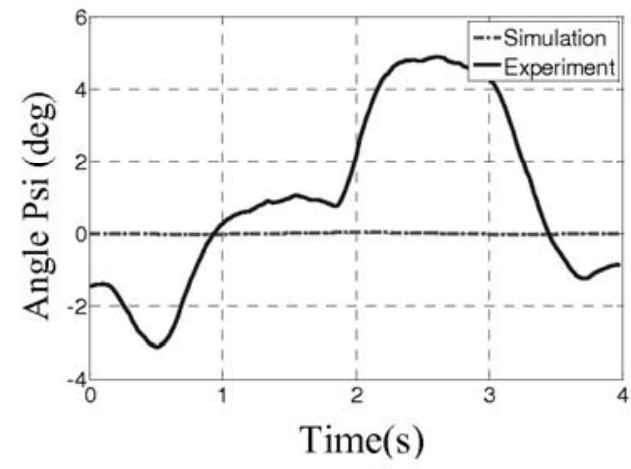

(d)

Figure 12. Orientation of platform MP for two-legged motion:

(a) A view in 3D space; (b) Angle $\phi$; (c) Angle $\theta$; (d) Angle $\psi$.

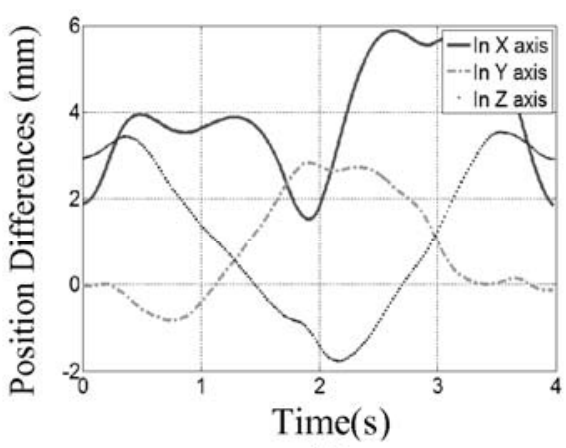

(a)

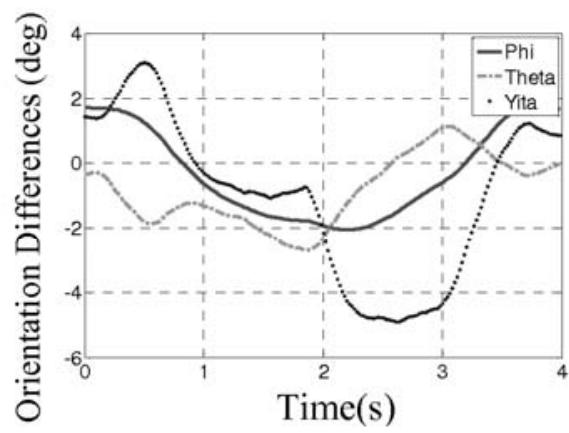

(b)

Figure 13. Differences between simulation and experimental results for two-legged motion: (a) Position differences; (b) Orientation angles differences 


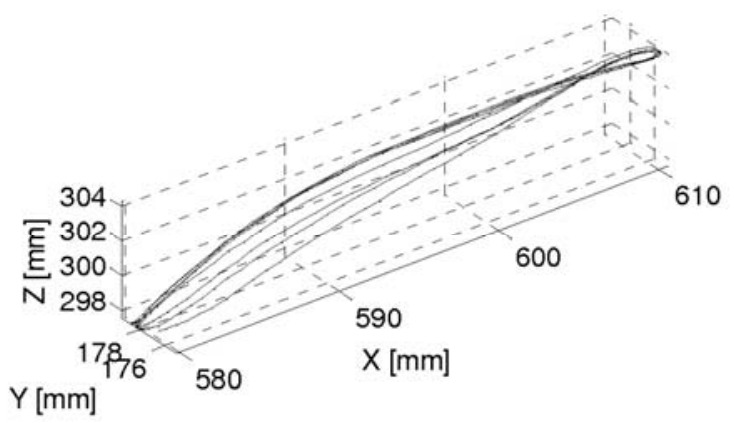

(a)

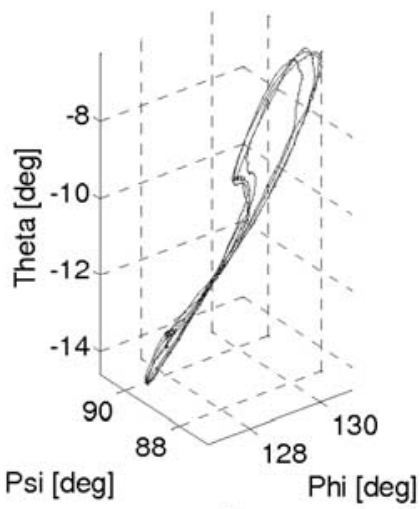

(b)

Figure 14. Experimental repeatability for four cycles of motion with two legs of CaPaMan 2bis: (a) Position of point $\mathrm{H}$ in Cartesian space; (b) Orientation of the MP platform in 3D space.

In Figures (15)-(17), results are reported for the third case of Table II. As shown in Figure 15(a), computed and measured trajectories of the endeffector point $C$ are shown in a Cartesian space. Corresponding trajectories inthe $X, Y$ and $Z$ axis are shown in Figures 15(b), (c) and (d). Figure 16 shows the orientation angles. The maximum position difference between computed and experimental results is $\pm 6 \mathrm{~mm}$ as shown in Figure 17(a). In four cycles of motion, Figures 18 (a) and (b) show the position and orientation repeatability for experiments with one leg of CaPaMan 2 bis.

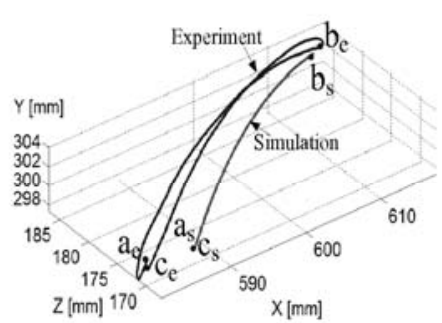

(a)

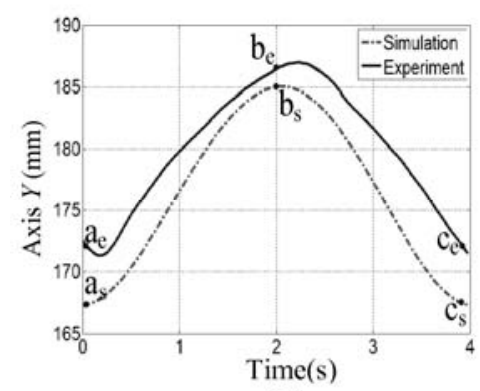

(c)

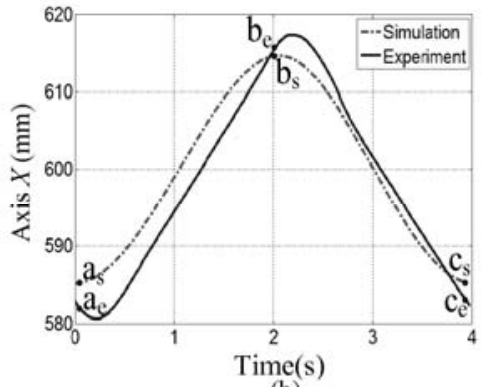

(b)

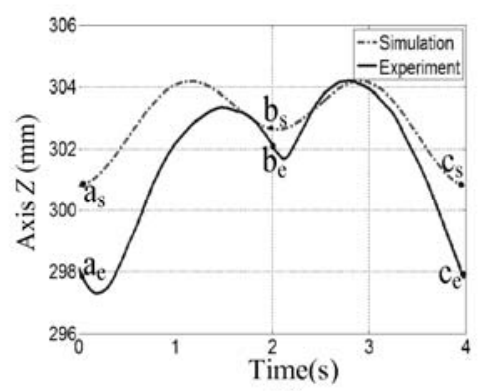

(d)

Figure 15. Position of the platform MP for one-legged motion: (a) Position of point $\mathrm{H}$ in Cartesian space; (b) Position in the $X$ axis; (c) Position in the $Y$ axis; (d) Position in the $Z$ axis. 


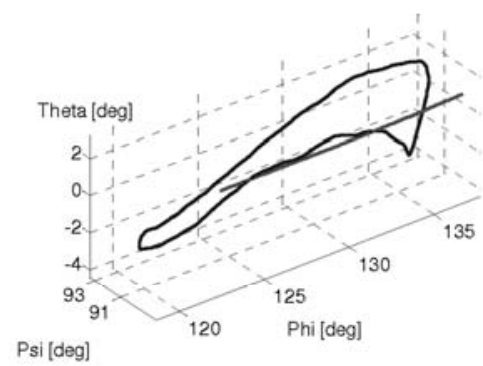

(a)

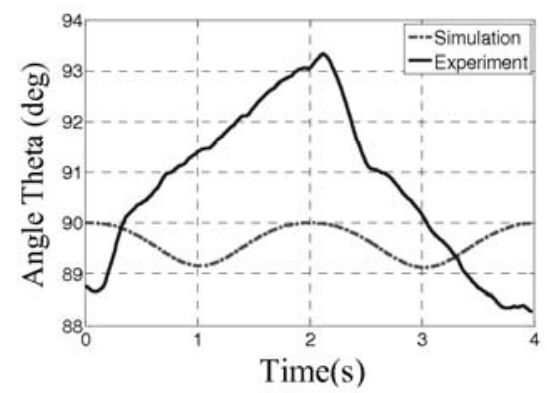

(c)

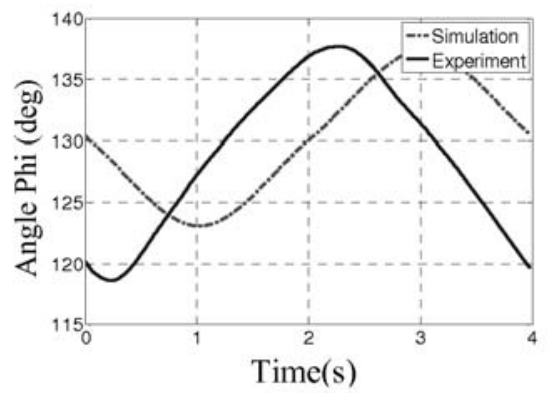

(b)

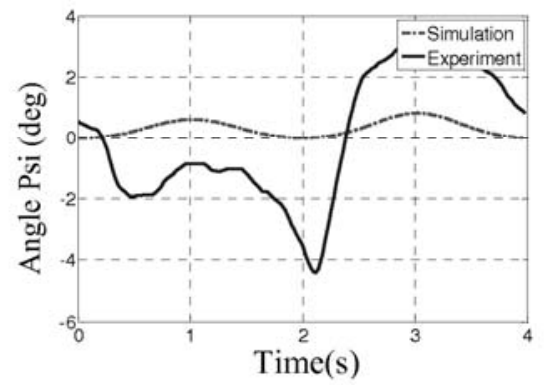

(d)

Figure 16. Orientation of platform MP for one-legged motion:

(a) A view in 3D space; (b) Angle $\phi$; (c) Angle $\theta$; (d) Angle $\psi$.

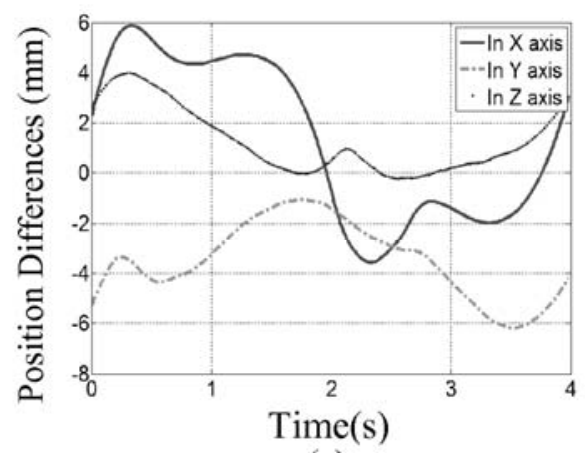

(a)

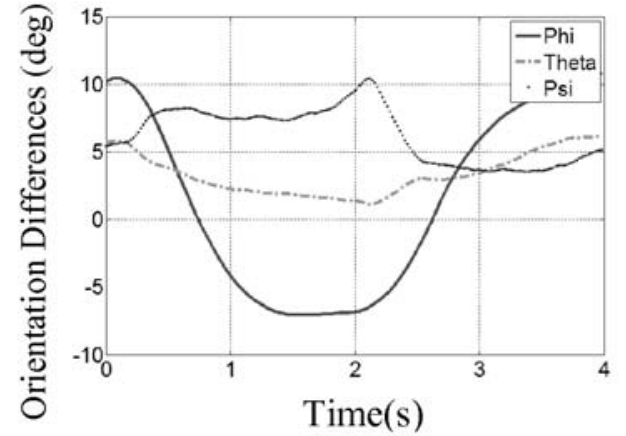

(b)

Figure 17. Differences between simulation and experimental results for one-legged motion:

(a) Position differences; (b) Orientation angles differences 


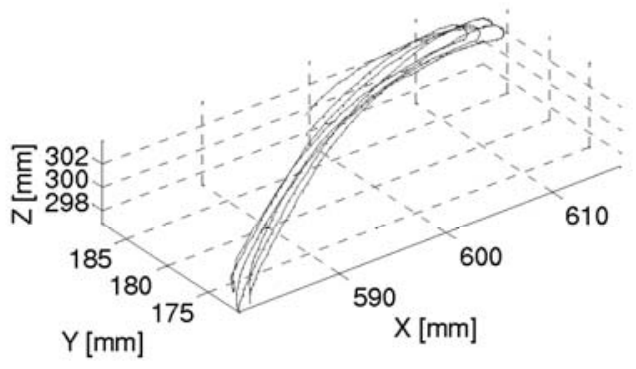

(a)

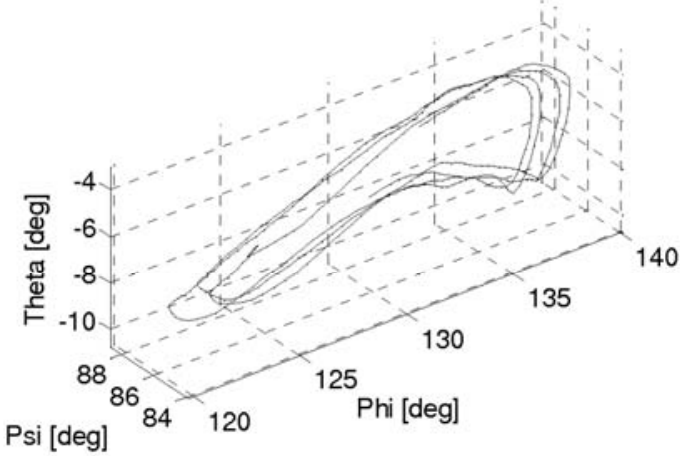

(b)

Figure 18. Experimental repeatability for four cycles of motion with one leg of CaPaMan 2bis: (a) Position of point $\mathrm{H}$ in Cartesian space; (b) Orientation of the MP platform in 3D space

\section{Conclusions}

A characterization on the CaPaMan 2bis operation was presented based on experimental tests and computer simulations. Several cases of study were considered to evaluate the operation performance of prototype CaPaMan 2bis for different motion conditions. Feasible position and orientation motions were determined for different operation modes. Kinematical design and operation of the prototype was characterized by feasible performance with smooth continuous actions and good repeatability ability. Comparison results show that different prescribed motions can be properly performed by the built prototype. In addition, the virtual model built in ADAMS environment can properly simulate the motion behavior of CaPaMan 2bis. Therefore, the formulated ADAMS model could be used as a motion simulator for CaPaMan 2 bis for future studies of enhancements or novel applications of the parallel architecture of CaPaMan 2bis.

\section{References}

[1] J. P. Merlet, Parallel Robots, Springer Publishers, Dordrecht, 2006.

[2] M. Ceccarelli, Fundamental of Mechanics of Robotic Manipulator, Kluwer Academic Publishers, Dordrecht, 2004.

[3] Datasheet of IRB 340 FlexPicker Parallel Arm Robot, ABB Robotics Inc. Available on line: www.abb.com/robots.

[4] Technical brochure of Adept Quattro ${ }^{\mathrm{TM}} \mathrm{s} 650 \mathrm{H}$ parallel robot, Adept Inc. Available on line: www.adept.com.

[5] Datasheet of IRB 940 Tricept Industrial Robot, ABB Robotics Inc. Available on line: www.abb.com/robots.

[6] ParalleIMIC- the parallel mechanism information center, available on line http://www.parallemic.org.

[7] J. P. Merlet, Still a long way to go on the road for parallel mechanisms, Proceedings of the 27th ASME Biennial Mechanism and Robotics Conference, 2002, Montreal. 
[8] M. Ceccarelli, A New 3 D.O.F. Parallel Spatial Mechanism, Mechanism and Machine Theory, Vol. 32, No. 8, 1997, pp. 895-902.

[9] E. Ottaviano, M. Ceccarelli, Optimal Design of CaPaMaN (Cassino Parallel Manipulator) with Prescribed Orientation Workspace, International Journal of Robotics, Vol. 20, 2002, pp. 159-166.

[10] M. Ceccarelli, G. Carbone, A Stiffness Analysis of CaPaMan (Cassino Parallel Manipulator), Mechanism and Machine Theory, Vol. 37, No. 5, 2002, pp. 427-439.

[11] M. Ceccarelli, P. M. Decio Fino, J. M. Jimenez, Dynamic Performance of CaPaMan by Numerical Simulations, Mechanism and Machine Theory, Vol. 37, 2002, pp. 241-266.

[12] J. C. Mendes-Carvalho, Ceccarelli, A Closed-Form Formulation for the Inverse Dynamics of a Cassino Parallel Manipulator, Multibody System Dynamics, Vol. 5, 2001, pp. 185-210.

[13] E. Ottaviano, M. Ceccarelli, An Application of a 3DOF Parallel Manipulator for Earthquake Simulations, IEEE Transactions on Mechatronics, Vol. 11, No. 2, 2006, pp. 240-146.

[14] G. Carbone, M. Ceccarelli, A Serial-Parallel Robotic Architecture for Surgical Tasks, Robotica, Vol. 23, 2005, pp. 345-354.

[15] N. E. Nava-Rodriguez, G. Carbone, M. Ceccarelli, CaPaMan 2bis as Trunk Module in CALUMA (CAssino Low-Cost hUMAnoid Robot), 2nd IEEE International Conference on Robotics, Automation and Mechatronics, RAM 06, 2006, pp. 347-352, Bangkok.

[16] G. Carbone, M. Ceccarelli, A Procedure for Experimental Evaluation of Cartesian Stiffness Matrix of Multibody Robotic Systems, 15th CISM-IFToMM Symposium on Robot Design, Dynamics and Control, Romansy, 2006, Montreal, Paper Rom04-24.

[17] G. Aguirre, M. Acevedo, G. Carbone, M. Ceccarelli, Kinematic and Dynamic Analyses of a 3 DOF Parallel Manipulator by Symbolic Formulations, Thematic Conference on Advances in Computational Multibody
Dynamics, ECCOMAS, 2003, Lisbon, Paper MB2003-010.

[18] LARM homepage. Available at http://webuser.unicas.it/weblarm/.

[19] E. E. Hernandez-Martinez, G. Carbone, C. LopezCajun, Operation Features of Milli-CaTraSys, Proceedings of 2-nd European Conference on Mechanism Science, EUCOMES'08, 2008, pp. 193-201, Cassino, Italy, September.

[20] F. Chang, Z-H. Lu, Dynamic Model of an Air Spring and Integration into a Vehicle Model. Proceedings of the Institution of Mechanical Engineers, Part D, Journal of Automobile Engineering, 222, 2008, pp.1813-1825.

[21] M. V. Blundell, The Modeling and Simulation of Vehicle Handling Part 1: Analysis Methods. Proceedings of the Institution of Mechanical Engineers, Part K, Journal of Multi-body Dynamics, 213, 1999, pp. 103118.

[22] N. Orlandea, Design of Parallel Kinematic Systems using the Planar Enveloping Algorithm and ADAMS Computer Program. Proceedings of the Institution of Mechanical Engineers, Part K, Journal of Multi-body Dynamics, 218, 2004.

\section{Acknowledgments}

The first author's PhD studies at LARM (2008 and 2009) have been supported by a grant from CONACYT (Mexican Science and Technology Council) and a cooperation program between the University of Cassino, Italy and the Universidad Autónoma de Querétaro, Mexico. He would like to express his gratitude to those institutions.

The second author's PhD studies at LARM (2008-2010) have been supported by a scholarship from the Chinese Scholarship Council (CSC), for which he is thankful. Authors would also like to thank CONACYT for supporting part of this project through grant $51410 \mathrm{Y}$. 


\section{Authors' Biography}

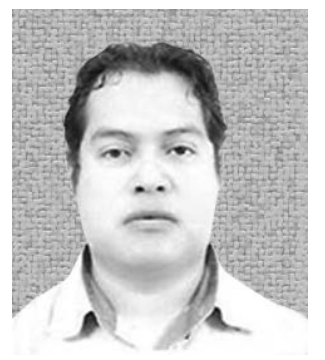

\section{Eusebio E. HERNÁNDEZ-MARTÍNEZ}

Eusebio E. Hernandez-Martínez was born in Queretaro, Mexico in 1978. He received his bachelor's degree in electronic engineering from Instituto Tecnológico de Celaya, Mexico in 2002. In 2004, he received his master's degree in instrumentation and automatic control at Universidad Autónoma de Querétaro (UAQ), México. Currently, he is a PhD candidate in the same university. He was a visiting PhD student at the University of Cassino, Italy in the years 2008 and 2009. His research interests include kinematics, calibration and error modeling of parallel robots. He is author of seven papers that have been published in proceedings of national and international conferences or in international journals. He is a member of SOMIM (Mexican Society of Mechanical Engineering).

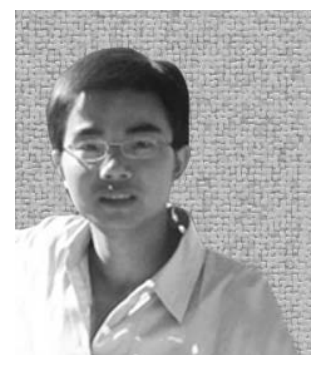

\section{Liang CONG-HUI}

Cong-Hui Liang was born in the city of Changsha in 1983 in China. He received his master's degree cum laude in mechatronics engineering in 2007 at the Behang University in Beijing and his bachelor's degree in automation at the Central-South University in Changsha in 2005. In 2008, he got a grant from the China Scholarship Council (CSC) for his three-year period of his PhD studies at the University of Cassino in Italy. From January 2008, he has been a Phd student at LARM: Laboratory of Robotics and Mechatronics at the University of Cassino. His research interests cover aspects of robotics with special attention to novel design and operation of humanoid robots. He also addresses research interests on manipulator workspace, robots control, and parallel manipulators. Until now, he has published four papers that have been presented at international conferences. 


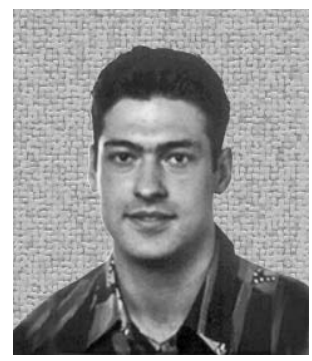

\section{Giuseppe CARBONE}

Giuseppe Carbone was born in Salerno in 1972. He got the degree with laude honors as mechanical engineer and the Ph.D. degree in mechanical engineering at the University of Cassino in 2000 and 2004, respectively. He is currently assistant professor at LARM: Laboratory of Robotics and Mechatronics in Cassino. He was visiting scholar at the Technical University of Braunschweig, Germany in 1999 and at the Humanoid Robotics Institute, Waseda University, Tokyo, Japan from 2002-2005 (partially supported by an Italian National Research Council CNR grant). His research interests cover aspects of mechanics of robots, mechanics of manipulation and grasp, mechanics of machinery. He is author and/or co-author of about 120 papers that have been published in proceedings of national and international conferences or in international journals. He is member of IEEE (the Institute of Electrical and Electronics Engineers).

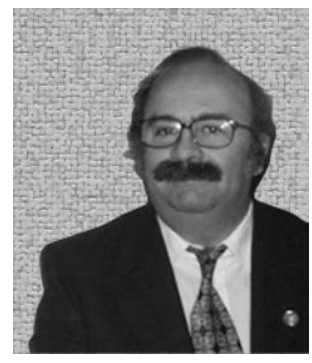

\section{Marco CECCARELLI}

Marco Ceccarelli is director of LARM, the Laboratory of Robotics and Mechatronics at the University of Cassino. He is full professor of mechanics of machinery and mechanisms. He is member of the IFToMM Commission for Robotics and the International Federation for the Promotion of Machine and Mechanism Science. He wrote the books 'Fundamentals of Mechanics of Robotic Manipulation', published by Kluwer/Springer in 2004, and 'Mecanismos', published by Trillas in Mexico in 2008. He was elected President of IFToMM for the term 2008-2011. His research interests cover aspects of theory of machines and mechanisms and mechanics of robots, with specific interest on analysis and design of workspace and manipulation, design of manipulators, legged robots, and grippers, history of TMM and mechanism design. He is author or co-author of more than four hundred papers which have been presented at conferences or published in national and international journals.

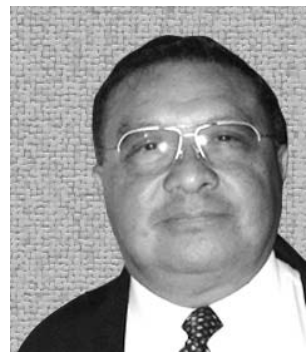

\section{Carlos S. LÓPEZ-CAJÚN}

Carlos S. López-Cajún received his BSME and MSME from UNAM in 1969 and 1977, respectively, and his PhD from Case Western Reserve University in 1982. He was professor at the UNAM, a visiting researcher at McGill University, and researcher at the Mexican Transportation Research Institute. Currently, he is professor at UAQ. His research focuses on simulation, robotics and mechanisms. He has published 20 journal papers, 30 conference extended publications and 2 textbooks on kinematics. He is a member of the Mexican Academy of Engineering and member of the IFToMM Executive Council and was elected as secretary general for the period 2008-2011. 